\title{
Existence theorems of generalized quasi-variational-like inequalities for pseudo-monotone type II operators
}

\author{
Mohammad SR Chowdhury ${ }^{1 *}$, Afrah AN Abdou² and Yeol Je Cho ${ }^{3 *}$
}

\section{"Correspondence:}

msrchowdhury@hotmail.com; showkat.chowdhry@umt.edu.pk; yjcho@gnu.ac.kr

'Department of Mathematics, University of Management and Technology (UMT), C-II, Johar Town, Lahore, 54770, Pakistan

${ }^{3}$ Department of Mathematics Education and RINS, Gyeongsang National University, Jinju 660-701, Korea

Full list of author information is available at the end of the article

\begin{abstract}
In this paper, we prove the existence results of solutions for a new class of generalized quasi-variational-like inequalities (GQVLI) for pseudo-monotone type II operators defined on compact sets in locally convex Hausdorff topological vector spaces. In obtaining our results on GQVLI for pseudo-monotone type II operators, we use Chowdhury and Tan's generalized version (Chowdhury and Cho in J. Inequal. Appl. 2012:79, 2012) of Ky Fan's minimax inequality (Fan in Inequalities, vol. III, pp.103-113, 1972) as the main tool.
\end{abstract}

Keywords: generalized quasi-variational-like inequalities; pseudo-monotone type II operators; locally convex Hausdorff topological vector spaces

\section{Introduction}

If $X$ is a nonempty set, then we denote by $2^{X}$ the family of all nonempty subsets of $X$ and by $\mathcal{F}(x)$ the family of all nonempty finite subsets of $X$. Let $E$ be a topological vector space over $\Phi, F$ be a vector space over $\Phi$ and $X$ be a nonempty subset of $E$. Let $\langle\cdot, \cdot\rangle: F \times E \rightarrow \Phi$ be a bilinear functional. Throughout this paper, $\Phi$ denotes either the real field $\mathbb{R}$ or the complex field $\mathbb{C}$.

For each $x_{0} \in E$, each nonempty subset $A$ of $E$ and each $\epsilon>0$, let $W\left(x_{0} ; \epsilon\right):=\{y \in F$ : $\left.\left|\left\langle y, x_{0}\right\rangle\right|<\epsilon\right\}$ and $U(A ; \epsilon):=\left\{y \in F: \sup _{x \in A}|\langle y, x\rangle|<\epsilon\right\}$. Let $\sigma\langle F, E\rangle$ be the (weak) topology on $F$ generated by the family $\{W(x ; \epsilon): x \in E, \epsilon>0\}$ as a subbase for the neighborhood system at 0 and $\delta\langle F, E\rangle$ be the (strong) topology on $F$ generated by the family $\{U(A ; \epsilon): A$ is a nonempty bounded subset of $E$ and $\epsilon>0\}$ as a base for the neighborhood system at 0 . We note then that $F$, when equipped with the (weak) topology $\sigma\langle F, E\rangle$ or the (strong) topology $\delta\langle F, E\rangle$, becomes a locally convex topological vector space which is not necessarily Hausdorff. But, if the bilinear functional $\langle\cdot, \cdot\rangle: F \times E \rightarrow \Phi$ separates points in $F$, i.e., for each $y \in F$ with $y \neq 0$, there exists $x \in E$ such that $\langle y, x\rangle \neq 0$, then $F$ also becomes Hausdorff. Furthermore, for any net $\left\{y_{\alpha}\right\}_{\alpha \in \Gamma}$ in $F$ and $y \in F$,

(a) $y_{\alpha} \rightarrow y$ in $\sigma\langle F, E\rangle$ if and only if $\left\langle y_{\alpha}, x\right\rangle \rightarrow\langle y, x\rangle$ for each $x \in E$;

(b) $y_{\alpha} \rightarrow y$ in $\delta\langle F, E\rangle$ if and only if $\left\langle y_{\alpha}, x\right\rangle \rightarrow\langle y, x\rangle$ uniformly for each $x \in A$, where $A$ is a nonempty bounded subset of $E$.

Suppose that, for the sets $X, E$ and $F$ mentioned above, $S: X \rightarrow 2^{X}$ and $T: X \rightarrow 2^{F}$ are two set-valued mappings. We now introduce below a slightly modified definition of the 
generalized quasi-variational inequality in infinite dimensional spaces given by Shih and Tan in [1]:

Find $\hat{y} \in S(\hat{y})$ and $\hat{w} \in T(\hat{y})$ such that

$$
\operatorname{Re}\langle\hat{w}, \hat{y}-x\rangle \leq 0
$$

for all $x \in S(\hat{y})$.

Now, we state the following definition which is a slightly corrected version of the corresponding definition given in [2]. Please note that there were typos in Definition 1.1 in [2].

Definition 1.1 Let the sets $X, E$ and $F$ and the mappings $S$ and $T$ be as defined above. Let $\eta: X \times X \rightarrow E$ be a single-valued mapping and $h: X \times X \rightarrow \mathbb{R}$ be a real-valued function. Then the generalized quasi-variational-like inequality problem is defined as follows: Find $\hat{y} \in S(\hat{y})$ and $\hat{w} \in T(\hat{y})$ such that

$$
\operatorname{Re}\langle\hat{w}, \eta(\hat{y}, x)\rangle+h(\hat{y}, x) \leq 0
$$

for all $x \in S(\hat{y})$.

For more results related to the generalized quasi-variational-like inequality problems, we refer to [3-6] and the references therein.

The following definition given in [7] is a slight modification of demi-operators defined in [8] and of pseudo-monotone type II operators defined in [9] (see also [10]).

Definition 1.2 Let $X$ be a nonempty subset of a topological vector space $E$ over $\Phi, F$ be a vector space over $\Phi$ which is equipped with $\sigma\langle F, E\rangle$-topology, where $\langle\cdot, \cdot\rangle: F \times E \rightarrow \Phi$ is a bilinear functional. Let $h: X \times X \rightarrow \mathbb{R}, \eta: X \times X \rightarrow E$ and $T: X \rightarrow 2^{F}$ be three mappings. Then $T$ is said to be:

(1) an $(\eta, h)$-pseudo-monotone type II (respectively, a strongly $(\eta, h)$-pseudo-monotone type II) operator if, for each $y \in X$ and every net $\left\{y_{\alpha}\right\}_{\alpha \in \Gamma}$ in $X$ converging to $y$ (respectively, weakly to $y$ ) with

$$
\limsup _{\alpha}\left[\inf _{u \in T(y)} \operatorname{Re}\left\langle u, \eta\left(y_{\alpha}, y\right)\right\rangle+h\left(y_{\alpha}, y\right)\right] \leq 0
$$

we have

$$
\begin{aligned}
& \underset{\alpha}{\limsup }\left[\inf _{u \in T(x)} \operatorname{Re}\left\langle u, \eta\left(y_{\alpha}, x\right)\right\rangle+h\left(y_{\alpha}, x\right)\right] \\
& \geq \inf _{u \in T(x)} \operatorname{Re}\langle u, \eta(y, x)\rangle+h(y, x)
\end{aligned}
$$

for all $x \in X$;

(2) an $h$-pseudo-monotone type II operator (respectively, a strongly h-pseudo-monotone type II operator) if $T$ is an $(\eta, h)$-pseudo-monotone type II operator with $\eta(x, y)=x-y$ and, for some $h^{\prime}: X \rightarrow \mathbb{R}, h(x, y)=h^{\prime}(x)-h^{\prime}(y)$ for all $x, y \in X$.

Note that, if $F=E^{*}$, the topological dual space of $E$, then the notions of $h$-pseudomonotone type II operators coincide with those in [8]. 
Pseudo-monotone type II operators were first introduced by Chowdhury in [8] with a slight variation in the name of this operator. Later, these operators were renamed as pseudo-monotone type II operators by Chowdhury in [9].

Next, we shall state and prove the following lemma which provides a numerous collection of $(\eta, h)$-pseudo-monotone type II and strongly $(\eta, h)$-pseudo-monotone type II operators.

Lemma 1.1 Let $E$ be a topological vector space and $X$ be a nonempty bounded subset of $E$. Let $T: X \rightarrow 2^{E^{*}}$ be an operator such that each $T(x)$ is strongly compact. Suppose that $h$ : $X \times X \rightarrow \mathbb{R}$ is a real-valued function such that, for each $y \in X, h(\cdot, y)$ is continuous and $h(X \times X)$ is bounded. Let $\eta: X \times X \rightarrow E$ be a continuous mapping. Suppose further that the operator $T$ is a continuous mapping from the relative weak topology on $X$ to the weak* topology on $E^{*}$. Then $T$ is both an $(\eta, h)$-pseudo-monotone type II and a strongly $(\eta, h)$ pseudo-monotone type II operator.

Proof Suppose that $\left\{y_{\alpha}\right\}_{\alpha \in \Gamma}$ is a net in $X$ and $y \in X$ with $y_{\alpha} \rightarrow y$ (respectively, $y_{\alpha} \rightarrow y$ weakly) and that

$$
\limsup _{\alpha}\left[\inf _{u \in T(y)} \operatorname{Re}\left\langle u, \eta\left(y_{\alpha}, y\right)\right\rangle+h\left(y_{\alpha}, y\right)\right] \leq 0 .
$$

Let $x \in X$ be arbitrarily fixed. Then, using the continuity of $h(\cdot, y), \eta$ and $T$, we obtain the following:

$$
\begin{aligned}
& \limsup _{\alpha}\left[\inf _{u \in T(x)} \operatorname{Re}\left\langle u, \eta\left(y_{\alpha}, x\right)\right\rangle+h\left(y_{\alpha}, x\right)\right] \\
& \geq \limsup _{\alpha}\left[\inf _{u \in T(x)} \operatorname{Re}\left\langle u, \eta\left(y_{\alpha}, x\right)\right\rangle\right]+\liminf _{\alpha} h\left(y_{\alpha}, x\right) \\
& \quad=\inf _{u \in T(x)} \operatorname{Re}\langle u, \eta(y, x)\rangle+h(y, x)
\end{aligned}
$$

for all $x \in X$. Consequently, $T$ is both an $(\eta, h)$-pseudo-monotone type II and a strongly $(\eta, h)$-pseudo-monotone type II operator.

The above lemma will, therefore, provide ample examples for our main results in Theorems 3.1 and 3.2 given in Section 3.

In this paper, we obtain some general theorems on solutions for a new class of generalized quasi-variational-like inequalities for pseudo-monotone type II operators defined on compact sets in topological vector spaces. In obtaining our results, we shall mainly use the following generalized version of Ky Fan's minimax inequality [11] due to Chowdhury and Tan which was stated and proved as Theorem 2.1 in [12] and is a slight modification of Theorem 1 in [13].

Theorem 1.2 Let $E$ be a Hausdorff topological vector space and $X$ be a nonempty convex subset of E. Let $h: X \times X \rightarrow \mathbb{R}$ and $\phi: X \times X \rightarrow \mathbb{R} \cup\{-\infty,+\infty\}$ be the mappings such that

(a) for each $A \in \mathcal{F}(X)$ and fixed $x \in \operatorname{co}(A), y \mapsto \phi(x, y)$ is lower semi-continuous on $\operatorname{co}(A)$;

(b) for each $A \in \mathcal{F}(X)$ and $y \in \operatorname{co}(A), \min _{x \in A}[\phi(x, y)+h(y, x)] \leq 0$; 
(c) for each fixed $x \in X, y \mapsto h(x, y)$ is lower semi-continuous and concave on $X$, and $h(x, x)=0 ;$

(d) for each $A \in \mathcal{F}(X)$ and each pair of points $x, y \in \operatorname{co}(A)$ such that every net $\left\{y_{\alpha}\right\}_{\alpha \in \Gamma}$ in $X$ converging to $y$ with $\phi\left(t x+(1-t) y, y_{\alpha}\right)+h\left(y_{\alpha}, t x+(1-t) y\right) \leq 0$ for all $\alpha \in \Gamma$ and all $t \in[0,1]$, we have $\phi(x, y)+h(y, x) \leq 0$;

(e) there exist a nonempty closed and compact subset $K$ of $X$ and $x_{0} \in K$ such that $\phi\left(x_{0}, y\right)+h\left(y, x_{0}\right)>0$ for all $y \in X \backslash K$.

Then there exists $\hat{y} \in K$ such that $\phi(x, \hat{y})+h(\hat{y}, x) \leq 0$ for all $x \in X$.

Proof For the proof, we refer to [12].

Definition 1.3 A function $\phi: X \times X \rightarrow \mathbb{R} \cup\{ \pm \infty\}$ is said to be 0 -diagonally concave (in short, 0 -DCV) in the second argument [14] if, for any finite set $\left\{x_{1}, \ldots, x_{n}\right\} \subset X$ and $\lambda_{i} \geq 0$ with $\sum_{i=1}^{n} \lambda_{i}=1$, we have $\sum_{i=1}^{n} \lambda_{i} \phi\left(y, x_{i}\right) \leq 0$, where $y=\sum_{i=1}^{n} \lambda_{i} x_{i}$.

Now, we state the following definition given in [15].

Definition 1.4 Let $X, E, F$ be the sets defined before and $T: X \rightarrow 2^{F}, \eta: X \times X \rightarrow E$, $g: X \rightarrow E$ be mappings.

(1) The mappings $T$ and $\eta$ are said to have the 0 -diagonally concave relation (in short, 0 -DCVR) if the function $\phi: X \times X \rightarrow \mathbb{R} \cup\{ \pm \infty\}$ defined by

$$
\phi(x, y)=\inf _{w \in T(x)} \operatorname{Re}\langle w, \eta(x, y)\rangle
$$

is $0-\mathrm{DCV}$ in $y$.

(2) The mappings $T$ and $g$ are said to have the 0 -diagonally concave relation if $T$ and $\eta(x, y)=g(x)-g(y)$ have the 0-DCVR.

The following definition of upper hemi-continuity was given in [16]. For a more general definition, we refer to Definition 1 in [17].

Definition 1.5 Let $E$ be a topological vector space, $X$ be a nonempty subset of $E$ and $T: X \rightarrow 2^{E^{*}}$. Then $T$ is said to be upper hemi-continuous on $X$ if and only if, for each $p \in E$, the function $f_{p}: X \rightarrow \mathbb{R} \cup\{+\infty\}$ defined by

$$
f_{p}(z)=\sup _{u \in T(z)} \operatorname{Re}\langle u, p\rangle
$$

for each $z \in X$ is upper semi-continuous on $X$ (if and only if, for each $p \in E$, the function $g_{p}: X \rightarrow \mathbb{R} \cup\{-\infty\}$ defined by

$$
g_{p}(z)=\inf _{u \in T(z)} \operatorname{Re}\langle u, p\rangle
$$

for each $z \in X$ is lower semi-continuous on $X)$.

\section{Preliminaries}

Now, we present some preliminary results in this section. First, we state the following result which is Lemma 1 of Shih and Tan in [1]. 
Lemma 2.1 Let $X$ be a nonempty subset of a Hausdorff topological vector space $E$ and $S: X \rightarrow 2^{E}$ be an upper semi-continuous map such that $S(x)$ is a bounded subset of $E$ for each $x \in X$. Then, for each continuous linear functional $p$ on $E$, the mapping $f_{p}: X \rightarrow \mathbb{R}$ defined by

$$
f_{p}(y)=\sup _{x \in S(y)} \operatorname{Re}\langle p, x\rangle
$$

is upper semi-continuous, i.e., for each $\lambda \in \mathbb{R}$, the set $\left\{y \in X: f_{p}(y)=\sup _{x \in S(y)} \operatorname{Re}\langle p, x\rangle<\lambda\right\}$ is open in $X$.

The following result is Lemma 3 of Takahashi in [18] (see also Lemma 3 in [19]).

Lemma 2.2 Let $X$ and $Y$ be topological spaces, $f: X \rightarrow \mathbb{R}$ be non-negative and continuous and $g: Y \rightarrow \mathbb{R}$ be lower semi-continuous. Then the mapping $F: X \times Y \rightarrow \mathbb{R}$ defined by $F(x, y)=f(x) g(y)$ for all $(x, y) \in X \times Y$ is lower semi-continuous.

The following result, which was stated and proved as Lemma 2.2 in [12], follows from slight modification of Lemma 3 of Chowdhury and Tan given in [13].

Lemma 2.3 Let E be a Hausdorff topological vector space over $\Phi, A \in \mathcal{F}(E)$ and $X=\operatorname{co}(A)$, where $\operatorname{co}(A)$ denotes the convex hull of $A$. Let $F$ be a vector space over $\Phi$ and $\langle\cdot, \cdot\rangle: F \times E \rightarrow \phi$ be a bilinear functional such that $\langle\cdot, \cdot\rangle$ separates points in $F$. We equip $F$ with the $\sigma\langle F, E\rangle-$ topology. Suppose that, for each $w \in F, x \mapsto \operatorname{Re}\langle w, x\rangle$ is continuous. Let $\eta: X \times X \rightarrow E$ be continuous. Let $T: X \rightarrow 2^{F}$ be upper semi-continuous from $X$ into $2^{F}$ such that each $T(x)$ is $\sigma\langle F, E\rangle$-compact. Let $f: X \times X \rightarrow \mathbb{R}$ be defined by $f(x, y)=\inf _{w \in T(y)} \operatorname{Re}\langle w, \eta(y, x)\rangle$ for all $x, y \in X$. Suppose that $\langle\cdot, \cdot\rangle$ is continuous on the (compact) subset $\left[\bigcup_{y \in X} T(y)\right] \times \eta(X \times X)$ of $F \times E$. Then, for each fixed $x \in X, y \mapsto f(x, y)$ is lower semi-continuous on $X$.

For the completeness, we include the proof here given in [12].

Proof Let $\lambda \in \mathbb{R}$ be given and let $x \in X=\operatorname{co}(A)$ be arbitrarily fixed. Let $A_{\lambda}=\{y \in X$ : $f(x, y) \leq \lambda\}$. Suppose that $\left\{y_{\alpha}\right\}_{\alpha \in \Gamma}$ is a net in $A_{\lambda}$ and $y_{0} \in \operatorname{co}(A)=X$ such that $y_{\alpha} \rightarrow y_{0}$. Then, for each $\alpha \in \Gamma$,

$$
\lambda \geq f\left(x, y_{\alpha}\right)=\inf _{w \in T\left(y_{\alpha}\right)} \operatorname{Re}\left\langle w, \eta\left(y_{\alpha}, x\right)\right\rangle .
$$

Since $F$ is equipped with the $\sigma\langle F, E\rangle$-topology, for each $x \in E$, the function $w \mapsto \operatorname{Re}\langle w, x\rangle$ is continuous. Also, $\eta\left(y_{\alpha}, x\right) \rightarrow \eta\left(y_{0}, x\right)$ because $\eta(\cdot, x)$ is continuous. By the $\sigma\langle F, E\rangle-$ compactness of $T\left(y_{\alpha}\right)$, there exists $w_{\alpha} \in T\left(y_{\alpha}\right)$ such that

$$
\lambda \geq \inf _{w \in T\left(y_{\alpha}\right)} \operatorname{Re}\left\langle w, \eta\left(y_{\alpha}, x\right)\right\rangle=\operatorname{Re}\left\langle w_{\alpha}, \eta\left(y_{\alpha}, x\right)\right\rangle .
$$

Since $T$ is upper semi-continuous from $X=\operatorname{co}(A)$ to the $\sigma\langle F, E\rangle$-topology on $F, X$ is compact, and each $T(z)$ is $\sigma\langle F, E\rangle$-compact, $\bigcup_{z \in X} T(z)$ is also $\sigma\langle F, E\rangle$-compact by Proposition 3.1.11 of Aubin and Ekeland [20]. Thus there is a subnet $\left\{w_{\alpha^{\prime}}\right\}_{\alpha^{\prime} \in \Gamma^{\prime}}$ of $\left\{w_{\alpha}\right\}_{\alpha \in \Gamma}$ and $w_{0} \in \bigcup_{z \in X} T(z)$ such that $w_{\alpha^{\prime}} \rightarrow w_{0}$ in the $\sigma\langle F, E\rangle$-topology. Again, as $T$ is upper semicontinuous with the $\sigma\langle F, E\rangle$-closed values, $w_{0} \in T\left(y_{0}\right)$. 
Suppose that $A=\left\{a_{1}, a_{2}, \ldots, a_{n}\right\}$ and let $t_{1}, t_{2}, \ldots, t_{n} \geq 0$ with $\sum_{i=1}^{n} t_{i}=1$ such that $y_{0}=$ $\sum_{i=1}^{n} t_{i} a_{i}$. For each $\alpha^{\prime} \in \Gamma$, let $t_{1}^{\alpha^{\prime}}, t_{2}^{\alpha^{\prime}}, \ldots, t_{n}^{\alpha^{\prime}} \geq 0$ with $\sum_{i=1}^{n} t_{i}^{\alpha^{\prime}}=1$ such that $y_{\alpha^{\prime}}=\sum_{i=1}^{n} t_{i}^{\alpha^{\prime}} a_{i}$. Since $E$ is Hausdorff and $y_{\alpha^{\prime}} \rightarrow y_{0}$, we must have $t_{i}^{\alpha^{\prime}} \rightarrow t_{i}$ for each $i=1,2, \ldots, n$. Thus

$$
\begin{aligned}
\lambda & \geq \operatorname{Re}\left\langle w_{\alpha^{\prime}}, \eta\left(y_{\alpha^{\prime}}, x\right)\right\rangle=\operatorname{Re}\left\langle w_{\alpha^{\prime}}, \eta\left(\sum_{i=1}^{n} t_{i}^{\alpha^{\prime}} a_{i}, x\right)\right\rangle \\
& \rightarrow \operatorname{Re}\left\langle w_{0}, \eta\left(\sum_{i=1}^{n} t_{i} a_{i}, x\right)\right\rangle \\
& =\operatorname{Re}\left\langle w_{0}, \eta\left(y_{0}, x\right)\right\rangle \geq \inf _{w \in T\left(y_{0}\right)} \operatorname{Re}\left\langle w, \eta\left(y_{0}, x\right)\right\rangle \\
& =f\left(x, y_{0}\right),
\end{aligned}
$$

where (2.1) is true since $\eta(\cdot, x)$ is continuous on $X$ and $\langle\cdot, \cdot\rangle$ is continuous on the compact subset $\left[\bigcup_{y \in X} T(y)\right] \times \eta(X \times X)$ of $F \times E$. Hence $y_{0} \in A_{\lambda}$. Thus $A_{\lambda}$ is closed in $X=\operatorname{co}(A)$ for each $\lambda \in \mathbb{R}$. Therefore $y \mapsto f(x, y)$ is lower semi-continuous on $X$. This completes the proof.

By the slight modification of Lemma 4.2 in [16], we obtained the following result given in [7] as Lemma 2.3.

Lemma 2.4 Let $E$ be a topological vector over $\phi, X$ be a nonempty convex subset of $E$ and $F$ be a vector space over $\phi$ with the $\sigma\langle F, E\rangle$-topology such that, for each $w \in F$, the function $x \mapsto \operatorname{Re}\langle w, x\rangle$ is continuous. Let $T: X \rightarrow 2^{F}$ be upper hemi-continuous along line segments in $X$. Let $\eta: X \times X \rightarrow E$ be such that for each fixed $y \in X, \eta(\cdot, y)$ is continuous, and let $h: X \times X \rightarrow \mathbb{R}$ be a mapping such that, for each fixed $y \in X, h(\cdot, y)$ is lower semi-continuous on $\operatorname{co}(A)$ for each $A \in \mathcal{F}(X)$ and, for each fixed $x \in X, h(x, \cdot)$ is concave and $h(x, x)=0$ and $T, \eta$ have the 0-DCVR. Suppose that $\hat{y} \in X$ such that $\inf _{u \in T(x)} \operatorname{Re}\langle u, \eta(\hat{y}, x)\rangle \leq h(x, \hat{y})$ for all $x \in X$. Then

$$
\inf _{w \in T(\hat{y})} \operatorname{Re}\langle w, \eta(\hat{y}, x)\rangle \leq h(x, \hat{y})
$$

for all $x \in X$.

We need the following Kneser's minimax theorem in [21] (see also Aubin [14]).

Theorem 2.5 Let $X$ be a nonempty convex subset of a vector space and $Y$ be a nonempty compact convex subset of a Hausdorff topological vector space. Suppose that $f$ is a realvalued function on $X \times Y$ such that for each fixed $x \in X$, the map $y \mapsto f(x, y)$, i.e., $f(x, \cdot)$ is lower semi-continuous and convex on $Y$ and,for each fixed $y \in Y$, the mapping $x \mapsto f(x, y)$, i.e., $f(\cdot, y)$ is concave on $X$. Then

$$
\min _{y \in Y} \sup _{x \in X} f(x, y)=\sup _{x \in X} \min _{y \in Y} f(x, y)
$$

\section{Generalized quasi-variational-like inequalities}

In this section, we prove some existence theorems for the solutions to the generalized quasi-variational-like inequalities for pseudo-monotone type II operators $T$ with compact 
domain in locally convex Hausdorff topological vector spaces. Our results extend and/or generalize the corresponding results in [1].

First, we establish the following result.

Theorem 3.1 Let E be a locally convex Hausdorff topological vector space over $\Phi, X$ be a nonempty compact convex subset of $E$ and $F$ be a vector space over $\Phi$ with $\sigma\langle F, E\rangle$-topology, where $\langle\cdot, \cdot\rangle: F \times E \rightarrow \Phi$ is a bilinear functional separating points on $F$ such that, for each $w \in F$, the function $x \mapsto \operatorname{Re}\langle w, x\rangle$ is continuous. Let $S: X \rightarrow 2^{X}, T: X \rightarrow 2^{F}, \eta: X \times X \rightarrow E$ and $h: E \times E \rightarrow \mathbb{R}$ be the mappings such that

(a) $S$ is upper semi-continuous such that each $S(x)$ is closed and convex;

(b) $h(X \times X)$ is bounded;

(c) $T$ is an $(\eta, h)$-pseudo-monotone type II (respectively, a strongly $(\eta, h)$-pseudo-monotone type II) operator and is upper hemi-continuous along line segments in $X$ to the $\sigma\langle F, E\rangle$-topology on $F$ such that each $T(x)$ is $\sigma\langle F, E\rangle$-compact and convex and $T(X)$ is $\delta\langle F, E\rangle$-bounded;

(d) $T$ and $\eta$ have the $0-D C V R$ and $\eta$ is continuous;

(e) for each fixed $y \in X, x \mapsto h(x, y)$, i.e., $h(\cdot, y)$ is lower semi-continuous on $\operatorname{co}(A)$ for each $A \in \mathcal{F}(X)$ and, for each fixed $x \in X, h(x, \cdot)$ and $\eta(x, \cdot)$ are concave, $\eta(x, \cdot)$ is affine, $h(x, x)=0$ and $\eta(x, x)=0$;

(f) the set $\Sigma=\left\{y \in X: \sup _{x \in S(y)}\left[\inf _{u \in T(x)} \operatorname{Re}\langle u, \eta(y, x)\rangle+h(y, x)\right]>0\right\}$ is open in $X$;

(g) for each $A \in \mathcal{F}(X)$ and each $y \in \operatorname{co}(A)$, there exist $\bar{x} \in A$ and $\bar{u} \in T(\bar{x})$ such that

$$
\beta_{0}(y)[\operatorname{Re}\langle\bar{u}, \eta(y, \bar{x})\rangle+h(y, \bar{x})]+\sum_{p \in \operatorname{LF}(E)} \beta_{p}(y) \operatorname{Re}\langle p, y-\bar{x}\rangle \leq 0
$$

for any family $\left\{\beta_{0}, \beta_{p}: p \in \operatorname{LF}(E)\right\}$ of non-negative real-valued functions from $X$ into $[0,1]$, where $\operatorname{LF}(E)$ denotes the set of all continuous linear functionals on $E$;

(h) for each $A \in \mathcal{F}(X)$, the bilinear functional $\langle\cdot, \cdot\rangle$ is continuous over the compact subset $\left[\bigcup_{y \in \operatorname{co}(A)} T(y)\right] \times \eta(\operatorname{co}(A) \times \operatorname{co}(A))$ of $F \times E$.

Then there exists a point $\hat{y} \in X$ such that

(1) $\hat{y} \in S(\hat{y})$;

(2) there exists a point $\hat{w} \in T(\hat{y})$ with $\operatorname{Re}\langle\hat{w}, \eta(\hat{y}, x)\rangle+h(\hat{y}, x) \leq 0$ for all $x \in S(\hat{y})$.

Proof Step 1. Let us first show that there exists a point $\hat{y} \in X$ such that $\hat{y} \in S(\hat{y})$ and

$$
\sup _{x \in S(\hat{y})}\left[\inf _{u \in T(x)} \operatorname{Re}\langle u, \eta(\hat{y}, x)\rangle+h(\hat{y}, x)\right] \leq 0 .
$$

Now, we prove this by contradiction. So, we assume that, for each $y \in X$, either $y \notin S(y)$ or there exists $x \in S(y)$ such that

$$
\inf _{u \in T(x)} \operatorname{Re}\langle u, \eta(y, x)\rangle+h(y, x)>0,
$$

that is, for each $y \in X$, either $y \notin S(y)$ or $y \in \Sigma$. If $y \notin S(y)$, then, by a slight modification of a separation theorem for convex sets in locally convex Hausdorff topological vector spaces, there exists a continuous linear functional $p$ on $E$ such that

$$
\operatorname{Re}\langle p, y\rangle-\sup _{x \in S(y)} \operatorname{Re}\langle p, x\rangle>0
$$


For each $y \in X$, set

$$
\begin{aligned}
& \gamma(y):=\sup _{x \in S(y)}\left[\inf _{u \in T(x)} \operatorname{Re}\langle u, \eta(y, x)\rangle+h(y, x)\right], \\
& V_{0}:=\Sigma=\{y \in X: \gamma(y)>0\}
\end{aligned}
$$

and, for each continuous linear functional $p$ on $E$,

$$
V_{p}:=\left\{y \in X: \operatorname{Re}\langle p, y\rangle-\sup _{x \in S(y)} \operatorname{Re}\langle p, x\rangle>0\right\} .
$$

Then we have

$$
X=V_{0} \cup \bigcup_{p \in \operatorname{LF}(E)} V_{p}
$$

Since $V_{0}$ is open by hypothesis and each $V_{p}$ is open in $X$ by Lemma 2.1 (Lemma 1 in [19]), $\left\{V_{0}, V_{p}: p \in \mathrm{LF}(E)\right\}$ is an open covering for $X$. Since $X$ is compact, there exist $p_{1}, p_{2}, \ldots, p_{n} \in \mathrm{LF}(E)$ such that $X=V_{0} \cup \bigcup_{i=1}^{n} V_{p_{i}}$. For the simplicity of notation, let $V_{i}=V_{p_{i}}$ for $i=1,2, \ldots, n$. Let $\left\{\beta_{0}, \beta_{1}, \ldots, \beta_{n}\right\}$ be a continuous partition of unity on $X$ subordinated to the covering $\left\{V_{0}, V_{1}, \ldots, V_{n}\right\}$. Then $\beta_{0}, \beta_{1}, \ldots, \beta_{n}$ are continuous non-negative real-valued functions on $X$ such that $\beta_{i}$ vanishes on $X \backslash V_{i}$ for each $i=0,1, \ldots, n$ and $\sum_{i=0}^{n} \beta_{i}(x)=1$ for all $x \in X$. Note that, for each $y \in X$ and $A \in \mathcal{F}(X), x \mapsto h(x, y)$, i.e., $h(\cdot, y)$ is continuous on $\operatorname{co}(A)$ (see [22], Corollary 10.1.1). Define a function $\phi: X \times X \rightarrow \mathbb{R}$ by

$$
\begin{aligned}
\phi(x, y)= & \beta_{0}(y)\left[\min _{u \in T(x)} \operatorname{Re}\langle u, \eta(y, x)\rangle+h(y, x)\right] \\
& +\sum_{i=1}^{n} \beta_{i}(y) \operatorname{Re}\left\langle p_{i}, y-x\right\rangle
\end{aligned}
$$

for all $x, y \in X$. Then we have the following:

(I) Since $E$ is Hausdorff, for each $A \in \mathcal{F}(X)$ and fixed $x \in \operatorname{co}(A)$, the mapping

$$
y \mapsto \inf _{u \in T(x)} \operatorname{Re}\langle u, \eta(y, x)\rangle+h(y, x)
$$

is continuous on $\operatorname{co}(A)$ by Lemma 2.3 and the fact that $h$ is continuous on $\operatorname{co}(A)$, and so the mapping

$$
y \mapsto \beta_{0}(y)\left[\min _{u \in T(x)} \operatorname{Re}\langle u, \eta(y, x)\rangle+h(y, x)\right]
$$

is lower semi-continuous on $\operatorname{co}(A)$ by Lemma 2.2. Also, for each fixed $x \in X$,

$$
y \mapsto \sum_{i=1}^{n} \beta_{i}(y) \operatorname{Re}\left\langle p_{i}, y-x\right\rangle
$$

is continuous on $X$. Hence, for each $A \in \mathcal{F}(X)$ and fixed $x \in \operatorname{co}(A)$, the mapping $y \mapsto \phi(x, y)$ is lower semi-continuous on $\operatorname{co}(A)$. 
(II) Since $\beta_{0}, \beta_{1}, \ldots, \beta_{n}$ is a family of continuous non-negative real-valued functions on $X$ into $[0,1]$, by the hypothesis, for each $A \in \mathcal{F}(X)$ and each $y \in \operatorname{co}(A)$, there exist $\bar{x} \in A$ and $\bar{u} \in T(\bar{x})$ such that

$$
\beta_{0}(y)[\operatorname{Re}\langle\bar{u}, \eta(y, \bar{x})\rangle+h(y, \bar{x})]+\sum_{i=1}^{n} \beta_{i}(y) \operatorname{Re}\left\langle p_{i}, y-\bar{x}\right\rangle \leq 0
$$

Thus we have

$$
\min _{u \in T(x)}\left[\beta_{0}(y)(\operatorname{Re}\langle u, \eta(y, \bar{x})\rangle+h(y, \bar{x}))\right]+\sum_{i=1}^{n} \beta_{i}(y) \operatorname{Re}\left\langle p_{i}, y-\bar{x}\right\rangle \leq 0,
$$

i.e.,

$$
\beta_{0}(y)\left[\min _{u \in T(x)}(\operatorname{Re}\langle u, \eta(y, \bar{x})\rangle+h(y, \bar{x}))\right]+\sum_{i=1}^{n} \beta_{i}(y) \operatorname{Re}\left\langle p_{i}, y-\bar{x}\right\rangle \leq 0 .
$$

Therefore, we have

$$
\min _{x \in A}\left[\beta_{0}(y)\left(\min _{u \in T(x)}(\operatorname{Re}\langle u, \eta(y, x)\rangle+h(y, x))\right)+\sum_{i=1}^{n} \beta_{i}(y) \operatorname{Re}\left\langle p_{i}, y-x\right\rangle\right] \leq 0
$$

and so $\min _{x \in A} \phi(x, y) \leq 0$ for each $A \in \mathcal{F}(X)$ and $y \in \operatorname{co}(A)$.

(III) Suppose that $A \in \mathcal{F}(X), x, y \in \operatorname{co}(A)$ and $\left\{y_{\alpha}\right\}_{\alpha \in \Gamma}$ is a net in $X$ converging to $y$ (respectively, weakly to $y$ ) with $\phi\left(t x+(1-t) y, y_{\alpha}\right) \leq 0$ for all $\alpha \in \Gamma$ and all $t \in[0,1]$.

Case 1: $\beta_{0}(y)=0$. Since $\beta_{0}$ is continuous and $y_{\alpha} \rightarrow y$, we have $\beta_{0}\left(y_{\alpha}\right) \rightarrow \beta_{0}(y)=0$. Note that $\beta_{0}\left(y_{\alpha}\right) \geq 0$ for each $\alpha \in \Gamma$. Since $T(X)$ is strongly bounded and $\left\{y_{\alpha}\right\}_{\alpha \in \Gamma}$ is a bounded net, it follows that

$$
\underset{\alpha}{\lim \sup }\left[\beta_{0}\left(y_{\alpha}\right)\left(\min _{u \in T(x)} \operatorname{Re}\left\langle u, \eta\left(y_{\alpha}, x\right)\right\rangle+h\left(y_{\alpha}, x\right)\right)\right]=0 .
$$

Also, we have

$$
\beta_{0}(y)\left[\min _{u \in T(x)} \operatorname{Re}\langle u, \eta(y, x)\rangle+h(y, x)\right]=0 .
$$

Thus it follows from (3.1) that

$$
\begin{aligned}
& \limsup _{\alpha}\left[\beta_{0}\left(y_{\alpha}\right) \min _{u \in T(x)} \operatorname{Re}\left\langle u, \eta\left(y_{\alpha}, x\right)\right\rangle+h\left(y_{\alpha}, x\right)\right] \\
& +\sum_{i=1}^{n} \beta_{i}(y) \operatorname{Re}\left\langle p_{i}, y-x\right\rangle \\
& =\sum_{i=1}^{n} \beta_{i}(y) \operatorname{Re}\langle p, y-x\rangle \\
& =\beta_{0}(y)\left[\min _{u \in T(x)} \operatorname{Re}\langle u, \eta(y, x)\rangle+h(y, x)\right] \\
& +\sum_{i=1}^{n} \beta_{i}(y) \operatorname{Re}\langle p, y-x\rangle .
\end{aligned}
$$


When $t=1$, we have $\phi\left(x, y_{\alpha}\right) \leq 0$ for all $\alpha \in \Gamma$, i.e.,

$$
\begin{aligned}
& \beta_{0}\left(y_{\alpha}\right)\left[\min _{u \in T(x)} \operatorname{Re}\left\langle u, \eta\left(y_{\alpha}, x\right)\right\rangle+h\left(y_{\alpha}, x\right)\right] \\
& +\sum_{i=1}^{n} \beta_{i}\left(y_{\alpha}\right) \operatorname{Re}\left\langle p, y_{\alpha}-x\right\rangle \\
& \leq 0
\end{aligned}
$$

for all $\alpha \in \Gamma$. Therefore, by (3.3), we have

$$
\begin{aligned}
& \limsup _{\alpha}\left[\beta_{0}\left(y_{\alpha}\right)\left(\min _{u \in T(x)} \operatorname{Re}\left\langle u, \eta\left(y_{\alpha}, x\right)\right\rangle+h\left(y_{\alpha}, x\right)\right)\right] \\
& \quad+\underset{\alpha}{\liminf }\left[\sum_{i=1}^{n} \beta_{i}\left(y_{\alpha}\right) \operatorname{Re}\left\langle p, y_{\alpha}-x\right\rangle\right] \\
& \leq \underset{\alpha}{\limsup }\left[\beta_{0}\left(y_{\alpha}\right)\left(\min _{u \in T(x)} \operatorname{Re}\left\langle u, \eta\left(y_{\alpha}, x\right)\right\rangle+h\left(y_{\alpha}, x\right)\right)\right. \\
& \left.\quad+\sum_{i=1}^{n} \beta_{i}\left(y_{\alpha}\right) \operatorname{Re}\left\langle p, y_{\alpha}-x\right\rangle\right] \\
& \leq 0
\end{aligned}
$$

and so

$$
\begin{aligned}
\limsup _{\alpha} & {\left[\beta_{0}\left(y_{\alpha}\right)\left(\min _{u \in T(x)} \operatorname{Re}\left\langle u, \eta\left(y_{\alpha}, x\right)\right\rangle+h\left(y_{\alpha}, x\right)\right)\right] } \\
& +\sum_{i=1}^{n} \beta_{i}(y) \operatorname{Re}\langle p, y-x\rangle \\
\leq & 0 .
\end{aligned}
$$

Hence, by (3.2) and (3.4), we have $\phi(x, y) \leq 0$.

Case 2: $\beta_{0}(y)>0$. Since $\beta_{0}$ is continuous, $\beta_{0}\left(y_{\alpha}\right) \rightarrow \beta_{0}(y)$. Again since $\beta_{0}(y)>0$, there exists $\lambda \in \Gamma$ such that $\beta_{0}\left(y_{\alpha}\right)>0$ for all $\alpha \geq \lambda$.

When $t=0$, we have $\phi\left(y, y_{\alpha}\right) \leq 0$ for all $\alpha \in \Gamma$, i.e.,

$$
\beta_{0}\left(y_{\alpha}\right)\left[\min _{u \in T(y)} \operatorname{Re}\left\langle u, \eta\left(y_{\alpha}, y\right)\right\rangle+h\left(y_{\alpha}, y\right)\right]+\sum_{i=1}^{n} \beta_{i}\left(y_{\alpha}\right) \operatorname{Re}\left\langle p, y_{\alpha}-y\right\rangle \leq 0
$$

for all $\alpha \in \Gamma$, and so

$$
\begin{aligned}
\underset{\alpha}{\limsup }[ & {\left[\beta_{0}\left(y_{\alpha}\right)\left(\min _{u \in T(y)} \operatorname{Re}\left\langle u, \eta\left(y_{\alpha}, y\right)\right\rangle+h\left(y_{\alpha}, y\right)\right)\right.} \\
& \left.+\sum_{i=1}^{n} \beta_{i}\left(y_{\alpha}\right) \operatorname{Re}\left\langle p, y_{\alpha}-y\right\rangle\right] \\
\leq & 0
\end{aligned}
$$


Hence, by (3.5), we have

$$
\begin{aligned}
& \underset{\alpha}{\limsup }\left[\beta_{0}\left(y_{\alpha}\right)\left(\min _{u \in T(y)} \operatorname{Re}\left\langle u, \eta\left(y_{\alpha}, y\right)\right\rangle+h\left(y_{\alpha}, y\right)\right)\right] \\
& \quad+\liminf _{\alpha}\left[\sum_{i=1}^{n} \beta_{i}\left(y_{\alpha}\right) \operatorname{Re}\left\langle p, y_{\alpha}-y\right\rangle\right] \\
& \leq \underset{\alpha}{\leq} \limsup _{\alpha}\left[\beta_{0}\left(y_{\alpha}\right)\left(\min _{u \in T(y)} \operatorname{Re}\left\langle u, \eta\left(y_{\alpha}, y\right)\right\rangle+h\left(y_{\alpha}, y\right)\right)\right. \\
& \left.\quad+\sum_{i=1}^{n} \beta_{i}\left(y_{\alpha}\right) \operatorname{Re}\left\langle p, y_{\alpha}-y\right\rangle\right] \\
& \leq 0 .
\end{aligned}
$$

Since $\liminf _{\alpha}\left[\sum_{i=1}^{n} \beta_{i}\left(y_{\alpha}\right) \operatorname{Re}\left\langle p, y_{\alpha}-y\right\rangle\right]=0$, we have

$$
\underset{\alpha}{\lim \sup }\left[\beta_{0}\left(y_{\alpha}\right)\left(\min _{u \in T(y)} \operatorname{Re}\left\langle u, \eta\left(y_{\alpha}, y\right)\right\rangle+h\left(y_{\alpha}, y\right)\right)\right] \leq 0 .
$$

Since $\beta_{0}\left(y_{\alpha}\right)>0$ for all $\alpha \geq \lambda$, it follows that

$$
\begin{aligned}
& \beta_{0}(y) \limsup _{\alpha}\left[\min _{u \in T(y)} \operatorname{Re}\left\langle u, \eta\left(y_{\alpha}, y\right)\right\rangle+h\left(y_{\alpha}, y\right)\right] \\
& =\underset{\alpha}{\lim \sup }\left[\beta_{0}\left(y_{\alpha}\right)\left(\min _{u \in T(y)} \operatorname{Re}\left\langle u, \eta\left(y_{\alpha}, y\right)\right\rangle+h\left(y_{\alpha}, y\right)\right)\right] .
\end{aligned}
$$

Since $\beta_{0}(y)>0$, by (3.6) and (3.7), we have

$$
\limsup _{\alpha}\left[\min _{u \in T(y)} \operatorname{Re}\left\langle u, \eta\left(y_{\alpha}, y\right)\right\rangle+h\left(y_{\alpha}, y\right)\right] \leq 0 .
$$

Since $T$ is an $(\eta, h)$-pseudo-monotone type II (respectively, a strongly $(\eta, h)$-pseudomonotone type II) operator, we have

$$
\begin{gathered}
\limsup _{\alpha}\left[\min _{u \in T(x)} \operatorname{Re}\left\langle u, \eta\left(y_{\alpha}, x\right)\right\rangle+h\left(y_{\alpha}, x\right)\right] \\
\geq \min _{u \in T(x)} \operatorname{Re}\langle u, \eta(y, x)\rangle+h(y, x)
\end{gathered}
$$

for all $x \in X$. Since $\beta_{0}(y)>0$, we have

$$
\begin{aligned}
& \beta_{0}(y)\left[\limsup _{\alpha} \min _{u \in T(x)} \operatorname{Re}\left\langle u, \eta\left(y_{\alpha}, x\right)\right\rangle+h\left(y_{\alpha}, x\right)\right] \\
& \geq \beta_{0}(y)\left[\min _{u \in T(x)} \operatorname{Re}\langle u, \eta(y, x)\rangle+h(y, x)\right],
\end{aligned}
$$

and so

$$
\begin{aligned}
\beta_{0}(y) & {\left[\limsup _{\alpha} \min _{u \in T(x)} \operatorname{Re}\left\langle u, \eta\left(y_{\alpha}, x\right)\right\rangle+h\left(y_{\alpha}, x\right)\right] } \\
& +\sum_{i=1}^{n} \beta_{i}(y) \operatorname{Re}\langle p, y-x\rangle
\end{aligned}
$$




$$
\begin{aligned}
\geq & \beta_{0}(y)\left[\min _{u \in T(x)} \operatorname{Re}\langle u, \eta(y, x)\rangle+h(y, x)\right] \\
& +\sum_{i=1}^{n} \beta_{i}(y) \operatorname{Re}\langle p, y-x\rangle .
\end{aligned}
$$

When $t=1$, we have $\phi\left(x, y_{\alpha}\right) \leq 0$ for all $\alpha \in \Gamma$, i.e.,

$$
\beta_{0}\left(y_{\alpha}\right)\left[\min _{u \in T(x)} \operatorname{Re}\left\langle u, \eta\left(y_{\alpha}, x\right)\right\rangle+h\left(y_{\alpha}, x\right)\right]+\sum_{i=1}^{n} \beta_{i}\left(y_{\alpha}\right) \operatorname{Re}\left\langle p, y_{\alpha}-x\right\rangle \leq 0
$$

for all $\alpha \in \Gamma$ and so, by (3.8),

$$
\begin{aligned}
0 \geq & \limsup _{\alpha}\left[\beta_{0}\left(y_{\alpha}\right)\left(\min _{u \in T(x)} \operatorname{Re}\left\langle u, \eta\left(y_{\alpha}, x\right)\right\rangle+h\left(y_{\alpha}, x\right)\right)\right. \\
& \left.+\sum_{i=1}^{n} \beta_{i}\left(y_{\alpha}\right) \operatorname{Re}\left\langle p, y_{\alpha}-x\right\rangle\right] \\
\geq & \limsup _{\alpha}\left[\beta_{0}\left(y_{\alpha}\right)\left(\min _{u \in T(x)} \operatorname{Re}\left\langle u, \eta\left(y_{\alpha}, x\right)\right\rangle+h\left(y_{\alpha}, x\right)\right)\right] \\
& +\liminf _{\alpha}\left[\sum_{i=1}^{n} \beta_{i}\left(y_{\alpha}\right) \operatorname{Re}\left\langle p, y_{\alpha}-x\right\rangle\right] \\
= & \beta_{0}(y)\left[\lim _{\alpha} \sup \left(\min _{u \in T(x)} \operatorname{Re}\left\langle u, \eta\left(y_{\alpha}, x\right)\right\rangle+h\left(y_{\alpha}, x\right)\right)\right] \\
& +\sum_{i=1}^{n} \beta_{i}(y) \operatorname{Re}\langle p, y-x\rangle \\
\geq & \beta_{0}(y)\left[\min _{u \in T(x)} \operatorname{Re}\langle u, \eta(y, x)\rangle+h(y, x)\right] \\
& +\sum_{i=1}^{n} \beta_{i}(y) \operatorname{Re}\langle p, y-x\rangle .
\end{aligned}
$$

Hence we have $\phi(x, y) \leq 0$.

(IV) Since $X$ is a compact (respectively, weakly compact) subset of the Hausdorff topological vector space $E$, it is also closed. Now, if we take $K=X$, then, for any $x_{0} \in K=X$, we have $\phi\left(x_{0}, y\right)>0$ for all $y \in X \backslash K(=X \backslash X=\emptyset)$. Thus the hypothesis (d) of Theorem 1.2 is satisfied trivially. (If $T$ is a strongly $(\eta, h)$-quasi-pseudo-monotone type II operator, we equip $E$ with the weak topology.) Thus $\phi$ satisfies all the hypotheses of Theorem 1.2. Hence, by Theorem 1.2 , there exists a point $\hat{y} \in K=X$ such that $\phi(x, \hat{y}) \leq 0$ for all $x \in X$, i.e.,

$$
\beta_{0}(\hat{y})\left[\min _{u \in T(x)} \operatorname{Re}\langle u, \eta(\hat{y}, x)\rangle+h(\hat{y}, x)\right]+\sum_{i=1}^{n} \beta_{i}(\hat{y}) \operatorname{Re}\left\langle p_{i}, \hat{y}-x\right\rangle \leq 0
$$

for all $x \in X$.

If $\beta_{0}(\hat{y})>0$, then $\hat{y} \in V_{0}=\Sigma$ so that $\gamma(\hat{y})>0$. Choose $\hat{x} \in S(\hat{y}) \subset X$ such that

$$
\min _{u \in T(\hat{x})} \operatorname{Re}\langle u, \eta(\hat{y}, \hat{x})\rangle+h(\hat{y}, \hat{x}) \geq \gamma(\hat{y}) / 2>0
$$


Then it follows that

$$
\beta_{0}(\hat{y})\left[\min _{u \in T(\hat{x})} \operatorname{Re}\langle u, \eta(\hat{y}, \hat{x})\rangle+h(\hat{y}, \hat{x})\right]>0
$$

If $\beta_{i}(\hat{y})>0$ for each $i=1,2, \ldots, n$, then $\hat{y} \in V_{i}$ and hence

$$
\operatorname{Re}\left\langle p_{i}, \hat{y}\right\rangle>\sup _{x \in S(\hat{y})} \operatorname{Re}\left\langle p_{i}, x\right\rangle \geq \operatorname{Re}\left\langle p_{i}, \hat{x}\right\rangle
$$

and so $\operatorname{Re}\left\langle p_{i}, \hat{y}-\hat{x}\right\rangle>0$. Then we see that $\beta_{i}(\hat{y}) \operatorname{Re}\left\langle p_{i}, \hat{y}-\hat{x}\right\rangle>0$ whenever $\beta_{i}(\hat{y})>0$ for each $i=1,2, \ldots, n$. Since $\beta_{0}(\hat{y})>0$ or $\beta_{i}(\hat{y})>0$ for each $i=1,2, \ldots, n$, it follows that

$$
\phi(\hat{x}, \hat{y})=\beta_{0}(\hat{y})\left[\min _{u \in T(\hat{x})} \operatorname{Re}\langle u, \eta(\hat{y}, \hat{x})\rangle+h(\hat{y}, \hat{x})\right]+\sum_{i=1}^{n} \beta_{i}(\hat{y}) \operatorname{Re}\left\langle p_{i}, \hat{y}-\hat{x}\right\rangle>0,
$$

which contradicts (3.10). This contradiction proves Step 1. Hence we have shown that there exists a point $\hat{y} \in X$ such that $\hat{y} \in S(\hat{y})$ and

$$
\sup _{x \in S(\hat{y})}\left[\inf _{u \in T(x)} \operatorname{Re}\langle u, \eta(\hat{y}, x)\rangle+h(\hat{y}, x)\right] \leq 0 .
$$

Step 2. We need to show that

$$
\inf _{w \in T(\hat{y})} \operatorname{Re}\langle w, \eta(\hat{y}, x)\rangle \leq h(x, \hat{y})
$$

for all $x \in S(\hat{y})$.

From Step 1, we know that $\hat{y} \in S(\hat{y})$ which is a convex subset of $X$ and

$$
\inf _{u \in T(x)} \operatorname{Re}\langle u, \eta(\hat{y}, x)\rangle \leq h(x, \hat{y})
$$

for all $x \in S(\hat{y})$. Hence, applying Lemma 2.4 , we obtain

$$
\inf _{w \in T(\hat{y})} \operatorname{Re}\langle w, \eta(\hat{y}, x)\rangle \leq h(x, \hat{y})
$$

for all $x \in S(\hat{y})$.

Step 3. There exists a point $\hat{w} \in T(\hat{y})$ with $\operatorname{Re}\langle\hat{w}, \eta(\hat{y}, x)\rangle \leq h(x, \hat{y})$ for all $x \in S(\hat{y})$. From Step 2, we have

$$
\sup _{x \in S(\hat{y})}\left[\inf _{w \in T(\hat{y})} \operatorname{Re}\langle w, \eta(\hat{y}, x)\rangle+h(\hat{y}, x)\right] \leq 0,
$$

where $T(\hat{y})$ is a $\sigma\langle F, E\rangle$-compact convex subset of the Hausdorff topological vector space $(F, \sigma\langle F, E\rangle)$ and $S(\hat{y})$ is a convex subset of $X$.

Now, we define $f: S(\hat{y}) \times T(\hat{y}) \rightarrow \mathbb{R}$ by $f(x, w)=\operatorname{Re}\langle w, \eta(\hat{y}, x)\rangle+h(\hat{y}, x)$ for each $x \in S(\hat{y})$ and $w \in T(\hat{y})$. Then, for each fixed $x \in S(\hat{y})$, the mapping $w \mapsto f(x, w)$ is convex and continuous on $T(\hat{y})$ and, for each fixed $w \in T(\hat{y})$, the mapping $x \mapsto f(x, w)$ is concave on $S(\hat{y})$. 
So, we can apply Keneser's minimax theorem (Theorem 2.5) and obtain the following:

$$
\min _{w \in T(\hat{y})} \sup _{x \in S(\hat{y})}[\operatorname{Re}\langle w, \eta(\hat{y}, x)\rangle+h(\hat{y}, x)]=\sup _{x \in S(\hat{y})} \min _{w \in T(\hat{y})}[\operatorname{Re}\langle w, \eta(\hat{y}, x)\rangle+h(\hat{y}, x)]
$$

Hence, by (3.11), we obtain

$$
\min _{w \in T(\hat{y})} \sup _{x \in S(\hat{y})}[\operatorname{Re}\langle w, \eta(\hat{y}, x)\rangle+h(\hat{y}, x)] \leq 0
$$

Since $T(\hat{y})$ is compact, there exists $\hat{w} \in T(\hat{y})$ such that

$$
\operatorname{Re}\langle\hat{w}, \eta(\hat{y}, x)\rangle+h(\hat{y}, x) \leq 0
$$

for all $x \in S(\hat{y})$. This completes the proof.

Note that, if for each open subset $U$ of $X$ and for each $x, y \in U, \eta(x, y)=x-y$ and there exists $h^{\prime}: X \rightarrow \mathbb{R}$ such that $h(x, y)=h^{\prime}(x)-h^{\prime}(y)$; and if the mapping $S: X \rightarrow 2^{X}$ is, in addition, lower semi-continuous and, for each $y \in \Sigma, T$ is upper semi-continuous at some point $x$ in $S(y)$ with $\inf _{u \in T(x)} \operatorname{Re}\langle u, \eta(y, x)\rangle+h(y, x)>0$, then the set $\Sigma$ in Theorem 3.1 is always open in $X$, and so we obtain the following result.

Theorem 3.2 Let E be a locally convex Hausdorff topological vector space over $\Phi, X$ be a nonempty compact convex subset of $E$ and $F$ be a vector space over $\Phi$ with $\sigma\langle F, E\rangle$-topology, where $\langle\cdot, \cdot\rangle: F \times E \rightarrow \Phi$ is a bilinear functional separating points on $F$ such that, for each $w \in F$, the function $x \mapsto \operatorname{Re}\langle w, x\rangle$ is continuous. Let $S: X \rightarrow 2^{X}, T: X \rightarrow 2^{F}, \eta: X \times X \rightarrow E$ and $h: E \times E \rightarrow \mathbb{R}$ be the mappings such that

(a) $S$ is continuous such that each $S(x)$ is closed and convex;

(b) $h(X \times X)$ is bounded;

(c) $T$ is an $(\eta, h)$-pseudo-monotone type II (respectively, a strongly $(\eta, h)$-pseudo-monotone type II) operator and is upper hemi-continuous along line segments in $X$ to the $\sigma\langle F, E\rangle$-topology on $F$ such that each $T(x)$ is $\sigma\langle F, E\rangle$-compact and convex and $T(X)$ is $\delta\langle F, E\rangle$-bounded;

(d) T and $\eta$ have the 0-DCVR and $\eta$ is continuous;

(e) for each fixed $y \in X, x \mapsto h(x, y)$, i.e., $h(\cdot, y)$ is lower semi-continuous on $\operatorname{co}(A)$ for each $A \in \mathcal{F}(X)$ and, for each fixed $x \in X, h(x, \cdot)$ and $\eta(x, \cdot)$ are concave, $\eta(x, \cdot)$ is affine, $h(x, x)=0$ and $\eta(x, x)=0$;

(f) for each open subset $U$ of $X$ and $x, y \in U, \eta(x, y)=x-y$, and there exists $h^{\prime}: X \rightarrow \mathbb{R}$ such that $h(x, y)=h^{\prime}(x)-h^{\prime}(y)$;

(g) for each $y \in \Sigma=\left\{y \in X: \sup _{x \in S(y)}\left[\inf _{u \in T(x)} \operatorname{Re}\langle u, \eta(y, x)\rangle+h(y, x)\right]>0\right\}$, T is upper semi-continuous at some point $x_{0}$ in $S(y)$ with $\inf _{u \in T\left(x_{0}\right)} \operatorname{Re}\left\langle u, \eta\left(y, x_{0}\right)\right\rangle+h\left(y, x_{0}\right)>0$;

(h) for each $A \in \mathcal{F}(X)$ and $y \in \operatorname{co}(A)$, there exist $\bar{x} \in A$ and $\bar{u} \in T(\bar{x})$ such that

$$
\beta_{0}(y)[\operatorname{Re}\langle\bar{u}, \eta(y, \bar{x})\rangle+h(y, \bar{x})]+\sum_{p \in \operatorname{LF}(E)} \beta_{p}(y) \operatorname{Re}\langle p, y-\bar{x}\rangle \leq 0
$$

for any family $\left\{\beta_{0}, \beta_{p}: p \in \mathrm{LF}(E)\right\}$ of non-negative real-valued functions from $X$ into $[0,1]$ 
(i) for each $A \in \mathcal{F}(X)$, the bilinear functional $\langle\cdot, \cdot\rangle$ is continuous over the compact subset $\left[\bigcup_{y \in \operatorname{co}(A)} T(y)\right] \times \eta(\operatorname{co}(A) \times \operatorname{co}(A))$ of $F \times E$.

Then there exists a point $\hat{y} \in X$ such that

(1) $\hat{y} \in S(\hat{y})$;

(2) there exists a point $\hat{w} \in T(\hat{y})$ with $\operatorname{Re}\langle\hat{w}, \eta(\hat{y}, x)\rangle+h(\hat{y}, x) \leq 0$ for all $x \in S(\hat{y})$.

The proof is similar to the proof of Theorem 3.2 in [10]. For the completeness, we include the proof here.

Proof The proof follows from Theorem 3.1 if we can show that the set

$$
\Sigma=\left\{y \in X: \sup _{x \in S(y)}\left[\inf _{u \in T(x)} \operatorname{Re}\langle u, \eta(y, x)\rangle+h(y, x)\right]>0\right\}
$$

is open in $X$. To show that $\Sigma$ is open in $X$, we start as follows.

Let $y_{0} \in \Sigma$ be an arbitrary point. We show that there exists an open neighborhood $N_{0}$ of $y_{0}$ in $X$ such that $N_{0} \subset \Sigma$. Now, by hypothesis (g), $T$ is upper semi-continuous at some point $x_{0}$ in $S\left(y_{0}\right)$ with

$$
\inf _{u \in T\left(x_{0}\right)} \operatorname{Re}\left\langle u, \eta\left(y_{0}, x_{0}\right)\right\rangle+h\left(y_{0}, x_{0}\right)>0
$$

Let

$$
\alpha:=\inf _{u \in T\left(x_{0}\right)} \operatorname{Re}\left\langle u, \eta\left(y_{0}, x_{0}\right)\right\rangle+h\left(y_{0}, x_{0}\right)
$$

Thus $\alpha>0$. Again, let

$$
W:=\left\{w \in F: \sup _{z_{1}, z_{2} \in X}\left|\left\langle w, z_{1}-z_{2}\right\rangle\right|<\alpha / 6\right\} .
$$

Then $W$ is a strongly open neighborhood of 0 in $F$, and so $U_{1}:=T\left(x_{0}\right)+W$ is an open neighborhood of $T\left(x_{0}\right)$ in $F$. Since $T$ is upper semi-continuous at $x_{0}$, there exists an open neighborhood $V_{1}$ of $x_{0}$ in $X$ such that $T(x) \subset U_{1}$ for all $x \in V_{1}$. Since the mapping $x \mapsto$ $\inf _{u \in T\left(x_{0}\right)} \operatorname{Re}\left\langle u, \eta\left(x_{0}, x\right)\right\rangle+h\left(x_{0}, x\right)$ is continuous at $x_{0}$, there exists an open neighborhood $V_{2}$ of $x_{0}$ in $X$ such that

$$
\left|\inf _{u \in T\left(x_{0}\right)} \operatorname{Re}\left\langle u, \eta\left(x_{0}, x\right)\right\rangle+h\left(x_{0}, x\right)\right|<\frac{\alpha}{6}
$$

for all $x \in V_{2}$. Let $V_{0}:=V_{1} \cap V_{2}$. Then $V_{0}$ is an open neighborhood of $x_{0}$ in $X$. Since $x_{0} \in V_{0} \cap S\left(y_{0}\right) \neq \emptyset$ and $S$ is lower semi-continuous at $y_{0}$, there exists an open neighborhood $N_{1}$ of $y_{0}$ in $X$ such that $S(y) \cap V_{0} \neq \emptyset$ for all $y \in N_{1}$. Since the mapping $y \mapsto$ $\inf _{u \in T\left(x_{0}\right)} \operatorname{Re}\left\langle u, \eta\left(y, y_{0}\right)\right\rangle+h\left(y, y_{0}\right)$ is continuous at $y_{0}$, there exists an open neighborhood $N_{2}$ of $y_{0}$ in $X$ such that

$$
\left|\inf _{u \in T\left(x_{0}\right)} \operatorname{Re}\left\langle u, \eta\left(y, y_{0}\right)\right\rangle+h\left(y, y_{0}\right)\right|<\frac{\alpha}{6}
$$

for all $y \in N_{2}$. 
Let $N_{0}:=N_{1} \cap N_{2}$. Then $N_{0}$ is an open neighborhood of $y_{0}$ in $X$ such that for each $y_{1} \in N_{0}$, we have the following:

(a) $S\left(y_{1}\right) \cap V_{0} \neq \emptyset$ as $y_{1} \in N_{1}$; so we can choose any $x_{1} \in S\left(y_{1}\right) \cap V_{0}$;

(b) $\left|\inf _{u \in T\left(x_{0}\right)} \operatorname{Re}\left\langle u, \eta\left(y_{1}, y_{0}\right)\right\rangle+h\left(y_{1}, y_{0}\right)\right|<\frac{\alpha}{6}$ as $y_{1} \in N_{2}$;

(c) $T\left(x_{1}\right) \subset U_{1}=T\left(x_{0}\right)+W$ as $x_{1} \in V_{1}$;

(d) $\left|\inf _{u \in T\left(x_{0}\right)} \operatorname{Re}\left\langle u, \eta\left(x_{0}, x_{1}\right)\right\rangle+h\left(x_{0}, x_{1}\right)\right|<\frac{\alpha}{6}$ as $x_{1} \in V_{2}$.

Hence, using property (f) and (b)-(d), we can obtain the following by omitting the details:

$$
\begin{aligned}
& \inf _{u \in T\left(x_{1}\right)} \operatorname{Re}\left\langle u, \eta\left(y_{1}, x_{1}\right)\right\rangle+h\left(y_{1}, x_{1}\right) \\
& \geq \inf _{\left[u \in T\left(x_{0}\right)+W\right]} \operatorname{Re}\left\langle u, \eta\left(y_{1}, x_{1}\right)\right\rangle+h\left(y_{1}, x_{1}\right) \\
& \geq \inf _{u \in T\left(x_{0}\right)} \operatorname{Re}\left\langle u, \eta\left(y_{1}, x_{1}\right)\right\rangle+h\left(y_{1}, x_{1}\right) \\
& \quad+\inf _{u \in W} \operatorname{Re}\left\langle u, \eta\left(y_{1}, x_{1}\right)\right\rangle \\
& \geq \inf _{u \in T\left(x_{0}\right)} \operatorname{Re}\left\langle u, y_{1}-y_{0}\right\rangle+h^{\prime}\left(y_{1}\right)-h^{\prime}\left(y_{0}\right) \\
& \quad+\inf _{u \in T\left(x_{0}\right)} \operatorname{Re}\left\langle u, y_{0}-x_{0}\right\rangle+h^{\prime}\left(y_{0}\right)-h^{\prime}\left(x_{0}\right) \\
& \quad+\inf _{u \in T\left(x_{0}\right)} \operatorname{Re}\left\langle u, x_{0}-x_{1}\right\rangle+h^{\prime}\left(x_{0}\right)-h^{\prime}\left(x_{1}\right) \\
& \quad+\inf _{u \in W} \operatorname{Re}\left\langle u, y_{1}-x_{1}\right\rangle \\
& \geq \\
& -\frac{\alpha}{6}+\alpha-\frac{\alpha}{6}-\frac{\alpha}{6} \\
& =\frac{\alpha}{2}>0 .
\end{aligned}
$$

Consequently, we have

$$
\sup _{x \in S\left(y_{1}\right)}\left[\inf _{u \in T(x)} \operatorname{Re}\left\langle u, \eta\left(y_{1}, x\right)\right\rangle+h\left(y_{1}, x\right)\right]>0,
$$

since $x_{1} \in S\left(y_{1}\right)$. Hence $y_{1} \in \Sigma$ for all $y_{1} \in N_{0}$. Therefore, $y_{0} \in N_{0} \subset \Sigma$. But $y_{0}$ was arbitrary. Consequently, $\Sigma$ is open in $X$. Thus all the hypotheses of Theorem 3.1 are satisfied. Hence, the conclusion follows from Theorem 3.1. This completes the proof.

\section{Remark 3.1}

(1) Theorems 3.1 and 3.2 of this paper are further extensions of the results obtained in [10, Theorem 3.1] and in [10, Theorem 3.2], respectively, into generalized quasi-variational-like inequalities of $(\eta, h)$-pseudo-monotone type II operators on compact sets.

(2) In 1985, Shih and Tan [1] obtained results on generalized quasi-variational inequalities in locally convex topological vector spaces, and their results were obtained on compact sets where the set-valued mappings were either lower semi-continuous or upper semi-continuous. Our present paper is another extension of the original work in [1] using $(\eta, h)$-pseudo-monotone type II operators on compact sets. 
(3) The results in [10] were obtained on non-compact sets where one of the set-valued mappings is a pseudo-monotone type II operator which was defined first in [8] and later renamed as pseudo-monotone type II operator in [9]. Our present results are extensions of the results in [10] using an extension of the operators defined in [9] (and originally in [8]).

\section{Competing interests}

The authors declare that they have no competing interests.

\section{Authors' contributions}

The first author made the first draft of this paper with substantial contributions to conception and design. The second and third authors have been involved equally in drafting the manuscript or revising it critically for important intellectual content. All authors read and approved the final manuscript.

\section{Author details}

'Department of Mathematics, University of Management and Technology (UMT), C-II, Johar Town, Lahore, 54770, Pakistan. ${ }^{2}$ Department of Mathematics, King Abdulaziz University, Jeddah, 21589, Saudi Arabia. ${ }^{3}$ Department of Mathematics Education and RINS, Gyeongsang National University, Jinju 660-701, Korea.

\section{Acknowledgements}

The second author was supported by the Deanship of Scientific Research (DSR), King Abdulaziz University, under grant No. 31-130-35-HiCi. The third author was supported by Basic Science Research Program through the National Research Foundation of Korea (NRF) funded by the Ministry of Science, ICT and Future Planning (2014R1A2A2A01002100).

Received: 12 March 2014 Accepted: 14 October 2014 Published: 05 Nov 2014

\section{References}

1. Shih, M-H, Tan, K-K: Generalized quasi-variational inequalities in locally convex topological vector spaces. J. Math. Anal. Appl. 108, 333-343 (1985)

2. Chowdhury, MSR, Cho, YJ: Existence theorems of generalized quasi-variational-like inequalities for $\eta$-h-pseudo-monotone type I operators on non-compact sets. J. Inequal. Appl. 2012, 79 (2012)

3. Cho, YJ, Lan, HY: A new class of generalized nonlinear multi-valued quasi-variational-like-inclusions with $H$-monotone mappings. Math. Inequal. Appl. 10, 389-401 (2007)

4. Chowdhury, MSR, Cho, YJ: Generalized bi-quasi-variational inequalities for quasi-pseudo-monotone type II operators on non-compact sets. J. Inequal. Appl. 2010, Article ID 237191 (2010)

5. Fang, YP, Cho, YJ, Huang, NJ, Kang, SM: Generalized nonlinear implicit quasi-variational-like inequalities for set-valued mappings in Banach spaces. Math. Inequal. Appl. 6, 331-337 (2003)

6. Lan, HY, Cho, YJ, Huang, NJ: Stability of iterative procedures for a class of generalized nonlinear quasi-variational-like inclusions involving maximal $\eta$-monotone mappings. In: Cho, YJ, Kim, JK, Kang, SM (eds.) Fixed Point Theory and Applications, vol. 6, pp. 107-116 (2006)

7. Chowdhury, MSR, Thompson, HB: Generalized variational-like inequalities for pseudo-monotone type II operators. Nonlinear Anal. 63, 321-330 (2005)

8. Chowdhury, MSR: Generalized variational inequalities for upper hemi-continuous and demi-operators with applications to fixed point theorems in Hilbert spaces. Serdica Math. J. 24, 163-178 (1998)

9. Chowdhury, MSR: The surjectivity of upper-hemicontinuous and pseudo-monotone type II operators in reflexive Banach spaces. Ganit 20, 45-53 (2000)

10. Chowdhury, MSR, Tarafdar, E: Existence theorems of generalized quasi-variational inequalities with upper hemi-continuous and demi-operators on non-compact sets. Math. Inequal. Appl. 2, 585-597 (1999)

11. Fan, K: A minimax inequality and applications. In: Shisha, O (ed.) Inequalities, vol. III, pp. 103-113. Academic Press, San Diego (1972)

12. Chowdhury, MSR, Tan, K-K: Generalized variational-like inequalities for pseudo-monotone type III operators. Cent. Eur. J. Math. 6(4), 526-536 (2008)

13. Chowdhury, MSR, Tan, K-K: Generalization of Ky Fan's minimax inequality with applications to generalized variational inequalities for pseudo-monotone operators and fixed theorems. J. Math. Anal. Appl. 204, 910-929 (1996)

14. Aubin, JP: Applied Functional Analysis. Wiley, New York (1979)

15. Ding, XP, Tarafdar, E: Generalized variational-like inequalities with pseudo-monotone set-valued mappings. Arch. Math. 74, 302-313 (2000)

16. Chowdhury, MSR, Tan, K-K: Generalized variational inequalities for quasi-monotone operators and applications. Bull. Pol. Acad. Sci., Math. 45, 25-54 (1997)

17. Chowdhury, MSR, Tan, K-K: Application of upper hemi-continuous operators on generalized bi-quasi-variational inequalities in locally convex topological vector spaces. Positivity 3, 333-344 (1999)

18. Takahashi, W: Nonlinear variational inequalities and fixed point theorems. J. Math. Soc. Jpn. 28, $166-181$ (1976)

19. Shih, M-H, Tan, K-K: Generalized bi-quasi-variational inequalities. J. Math. Anal. Appl. 143, 66-85 (1989)

20. Aubin, JP, Ekeland, I: Applied Nonlinear Analysis. Wiley, New York (1984)

21. Kneser, H: Sur un théorème fondamental de la théorie des jeux. C. R. Acad. Sci. Paris 234, 2418-2420 (1952)

22. Rockafeller, RT: Convex Analysis. Princeton University Press, Princeton (1970) 
10.1186/1029-242X-2014-449

Cite this article as: Chowdhury et al.: Existence theorems of generalized quasi-variational-like inequalities for pseudo-monotone type II operators. Journal of Inequalities and Applications 2014, 2014:449

Submit your manuscript to a SpringerOpen ${ }^{\circ}$ journal and benefit from:

- Convenient online submission

- Rigorous peer review

- Immediate publication on acceptance

Open access: articles freely available online

- High visibility within the field

- Retaining the copyright to your article

Submit your next manuscript at $\gg$ springeropen.com 